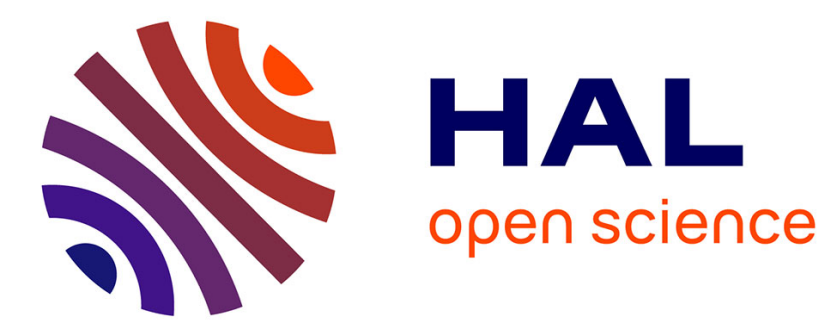

\title{
Image-based identification procedure of a crack propagation law
}

Florent Mathieu, François Hild, Stéphane Roux

\section{To cite this version:}

Florent Mathieu, François Hild, Stéphane Roux. Image-based identification procedure of a crack propagation law. Engineering Fracture Mechanics, 2013, 103, pp.48-59. hal-00848725

\section{HAL Id: hal-00848725 \\ https://hal.science/hal-00848725}

Submitted on 28 Jul 2013

HAL is a multi-disciplinary open access archive for the deposit and dissemination of scientific research documents, whether they are published or not. The documents may come from teaching and research institutions in France or abroad, or from public or private research centers.
L'archive ouverte pluridisciplinaire HAL, est destinée au dépôt et à la diffusion de documents scientifiques de niveau recherche, publiés ou non, émanant des établissements d'enseignement et de recherche français ou étrangers, des laboratoires publics ou privés. 


\title{
Image-based identification procedure of a crack propagation law
}

\author{
Florent Mathieu $^{a, c}$, François Hild ${ }^{a}$ and Stéphane Roux ${ }^{a}$ \\ ${ }^{a}$ Laboratoire de Mécanique et Technologie (LMT-Cachan) \\ ENS Cachan / CNRS / UPMC / PRES UniverSud Paris \\ 61 Avenue du Président Wilson, F-94235 Cachan Cedex, France \\ ${ }^{c}$ corresponding author. Email: florent.mathieu@lmt.ens-cachan.fr
}

\begin{abstract}
A novel methodology to identify a fatigue crack propagation law is proposed. It is based on an optimization scheme that directly interrogates the images from the sought law rather than using a sequence of fitting schemes. Two procedures are presented and tested first on artificial data with a known law, and second on an actual image series obtained from a fatigue test performed on a thin titanium sheet. The results demonstrate the ability of the proposed procedures to deal with inherently noisy data, and yet obtain an accurate propagation law. As a side result "admissible" time series of stress intensity factors and crack advance are obtained that fulfill the identified law and are constrained by the registration of actual images, rather than being simply filtered from the data in a post-processing step.
\end{abstract}

Key words: Digital image correlation, fatigue crack propagation, Paris' law, Uncertainty.

Email addresses: florent.mathieu@lmt.ens-cachan.fr (Florent Mathieu ${ }^{a, c}$, ), 


\section{Introduction}

The evaluation of crack propagation laws is important since a majority of engineering failures is due to fatigue. When assessing crack growth rates, one important source of variability is found to be related to the evaluation of the crack length [1]. Different indirect measurement techniques have been introduced in the past [2-5]. The direct current potential difference, the alternating current potential difference and the compliance method are the most commonly used methods. They are now standardized [6]. The minimum resolution should be less than $0.1 \mathrm{~mm}$ [7]. However, the techniques do not yield consistent results when dealing with closure phenomena [8]. Consequently, alternative approaches may be proposed.

Another route to follow the crack length is to use direct observations by resorting to optical means [4]. The most widely used technique consists in monitoring the propagating crack with optical microscopy. This approach needs a person to follow the crack propagation and decide when pictures are taken. The resolution in terms of crack opening is of the order of one pixel, which may lead to a very conservative estimate of the crack tip location since the crack opening displacement vanishes at the tip. Digital image correlation is an appealing technique that allows the user to process the picture to determine displacement fields [9], stress intensity factors from a subsequent analysis [10,11], or as a direct output of the correlation procedure $[12,13]$. The last approach will be used in the sequel. Very early on, it was recognized that DIC could give valuable information concerning for instance, crack tip opening angles after initial

francois.hild@lmt.ens-cachan.fr (François Hild $\left.{ }^{a}\right)$, stephane.roux@lmt.ens-cachan.fr (Stéphane Roux ${ }^{a}$ ). 
stable crack propagation [14]. Another advantage of the technique is that it detects crack closure and opening levels $[15,16,13]$, since sub-pixel resolutions are achieved to measure crack opening displacements $[15,16,12,17]$.

In many instances, the crack length is not the only parameter influencing crack propagation. The so-called T-stress $[18,19]$ has also an influence, for instance on the near stress-field for elasto-plastic materials [20,21]. This is also the case in fatigue crack growth [22-24]. For example, the scale parameter of Paris' law was altered to depend linearly on the T-stress [25]. Values of the T-stress are tabulated for standard sample geometries (e.g., CCT samples [26]) when the crack length is known. The experimental evaluation of the T-stress can be performed by resorting to strain gauges [27]. However, as the crack propagates, it is more difficult to implement. Another route is to use full-field measurements since the T-stress is one of the components of Williams' series [18]. It was shown that it is possible to measure and get good agreements with tabulated values when applied to a crack propagation test on commercially pure titanium [28].

A first procedure [28] was already developed to identify crack propagation by using an integrated approach to DIC (I-DIC). However, the crack propagation law was determined as a post-treatment of the various data (i.e., range of stress intensity factor, crack tip position). In particular, an automatic detection of the crack tip position was implemented. It is based upon the use of the first super-singular term of Williams' series [13,29]. In the present study, in order to minimize measurement uncertainties (i.e., to regularize the identification problem), the propagation law is postulated a priori. Consequently, the output of the measurement technique is directly the sought parameters. In the following analyses, a Paris' law $[30,31]$ is postulated. One issue addressed 
herein is the resolution of the technique. The key question of the relevance of the chosen propagation law is not directly investigated. The focus is put on the determination of the parameters with a specific frame of analysis. However, the final determination of the law also provides a residual difference between the estimated data and the value predicted by the model. Ideally, for an appropriate law, the resulting residual should be a white noise. Deviations from flat fluctuations can be interpreted as deficiencies of the proposed law (either inappropriate algebraic form, or missing influential parameters).

After a presentation of the principle of Integrated Digital Image Correlation in Section 2, the identification strategies are defined in Section 3. The two introduced methodologies are tested against artificial data in Section 4. Application to a fatigue test on a titanium thin sheet is presented and discussed in Section 5. Finally, Section 6 summarizes the main results, and discusses perspectives of the current work.

\section{Integrated Digital Image Correlation}

Global and integrated DIC $[12,13]$ is based on the conservation of gray level between a reference image $f$ and a deformed image $g$. It allows $f$ to be written as a function of $g$ and the displacement vector $\mathbf{u}$ for each position $x$ in the image

$$
f(\mathbf{x})=g(\mathbf{x}+\mathbf{u}(\mathbf{x}))
$$

From Equation (1), solving the registration problem (i.e., finding the best $\mathbf{u}$ field) is achieved by minimizing the sum of squared differences $R^{2}$ over the 
Region of Interest (ROI)

$$
R^{2}=\iint_{\mathrm{ROI}}[g(\mathbf{x}+\mathbf{u}(\mathbf{x}))-f(\mathbf{x})]^{2} \mathrm{~d} \mathbf{x}
$$

Integrated Digital Image Correlation (I-DIC) consists in using displacement fields that are mechanically meaningful. In the present case, Williams' series [18] is used. Contrary to classical approaches, the series is not bounded by the first term, which is related to rigid body translations. Super-singularterms are added to account for different phenomena such as a mis-positioning of the crack tip [32], confined plasticity [33]. The general form of Williams' series reads

$$
\mathbf{u}(z)=\sum_{n}\left[\omega_{n} \boldsymbol{\Omega}_{n}(z)+v_{n} \boldsymbol{\Upsilon}_{n}(z)\right]
$$

whose mode I part is

$$
\begin{aligned}
\boldsymbol{\Omega}_{n}(z) & =\frac{(-1)^{(1-n) / 2}}{2 \mu \sqrt{2 \pi}} r^{n / 2}\left[\kappa \exp \left(\frac{i n \theta}{2}\right)-\frac{n}{2} \exp \left(\frac{i(4-n) \theta}{2}\right)\right. \\
& \left.+\left((-1)^{n}+\frac{n}{2}\right) \exp \left(-\frac{i n \theta}{2}\right)\right]
\end{aligned}
$$

and mode II

$$
\begin{aligned}
\Upsilon_{n}(z) & =\frac{i(-1)^{(1-n) / 2}}{2 \mu \sqrt{2 \pi}} r^{n / 2}\left[\kappa \exp \left(\frac{i n \theta}{2}\right)+\frac{n}{2} \exp \left(\frac{i(4-n) \theta}{2}\right)\right. \\
& \left.+\left((-1)^{n}-\frac{n}{2}\right) \exp \left(-\frac{i n \theta}{2}\right)\right]
\end{aligned}
$$

where $z=r \exp (i \theta),(r, \theta)$ being the cylindrical coordinates with respect to the crack tip $(z=0), \mu$ Lamé's constant, and $\kappa$ Kolossov's constant $(\kappa=(3-\nu) /(1+\nu)$, where $\nu$ is Poisson's ratio of the material). The unknown parameters in the registration are therefore the amplitudes $\omega_{n}$ and $v_{n}$. Consequently, the outputs of an integrated DIC analysis that can be used are 
numerous $[12,13,28]$. I-DIC provides an estimate of $\Delta K$ that is unbiased and of low uncertainty [12]. It also provides a direct measurement of the image registration quality through the global residual $R^{2}(N)$ between the reference and the corrected deformed image at each studied instant of time (indexed by the number of cycles $N)$. Minimization of the cumulative residual $R_{t o t}=\sum_{N} R(N)$ could be a way to ensure that a global history of crack propagation sticks to the experimental data. However, the sensitivity of this quantity with respect to the sought parameters is not expected to allow for a very faithful determination of a crack propagation law. Hence, exploitation of this global residual has not been explored in the following.

I-DIC estimates the crack tip location, based on a criterion that is the vanishing of the amplitude of the first supersingular Williams' field $\omega_{-1}$. The latter allows the distance between the proposed crack tip for the analysis and the best position in the sense of Williams' series expansion to be estimated

$$
\Delta a=\frac{2 \omega_{-1}}{\omega_{1}}
$$

This way of estimating the crack tip position has revealed to be extremely precise and reliable both numerically [33] and experimentally [13,32,28]. Thus it is appealing to exploit this information in order to determine the crack growth law parameters.

\section{Identification of crack propagation law}

The crack length $a$ incremental law with respect to the number of cycles $N$ is written, in its most elementary form, as a function of the mode I Stress 
Intensity Factor Range (SIFR) $\Delta K$

$$
\frac{\mathrm{d} a}{\mathrm{~d} N}=\Psi(\Delta K)
$$

The purpose of the present paper is to design a technique to determine $\Psi$ function for a single experiment of fatigue crack propagation, during which a number of pictures is taken as the crack propagates, and samples different $\Delta K$ values. More precisely, a generic algebraic form of $\Psi$ is chosen a priori and its parameters are sought so that they match at best the experimental observations. One generic function often used in the literature is Paris' law [30,31]

$$
\Psi(\Delta K)=A(\Delta K)^{m}
$$

where amplitude $A$ and exponent $m$ are the $n_{p}=2$ parameters to be determined. Another popular form is

$$
\Psi(\Delta K)=B\left(\Delta K-\Delta K_{0}\right)^{p} \quad \text { when } \quad \Delta K \geq \Delta K_{0}
$$

where threshold $\Delta K_{0}$ accounts for crack closure phenomena [34,35]. The latter law involves $n_{p}=3$ parameters and contains the previous form (retrieved for $\Delta K_{0}=0$ ), hence it is naturally expected to provide a better fit to the experimental data, so that a significant reduction of the residuals has to be obtained in order to validate such a form. From now on, the parameters of $\Psi$ are called $\alpha_{i}$ for $i=1, \ldots, n_{p}$. The actual crack tip after $N$ cycles thus obeys

$$
a(N)=a\left(N_{0}\right)+\int_{N_{0}}^{N} \Psi(\Delta K(M)) \mathrm{d} M
$$

where $a\left(N_{0}\right)$ denotes the crack position after $N_{0}$ cycles. Two difficulties arise with the above expression. First, the crack growth law is generically non-linear, and second, the SIFR evolves with $N$ so that the integral over the cycle number can be seen as an integration with respect to $\Delta K$ with a non-uniform measure $1 /(\mathrm{d} \Delta K(N) / \mathrm{d} N)$, which is itself unknown. 
Many ways exist to determine the unknown parameters. In the ideal case, most of them would be equivalent. However, the difficulty to be faced here is the fact that DIC provide estimates for $a$ and $\Delta K$ that are not perfect but rather polluted by some noise [36]. The first and direct approach consists in calculating the crack growth rate with centered finite differences [37]. The direct use of $\mathrm{d} a / \mathrm{d} N$ is not a good strategy as errors in $a$ are drastically enhanced in the incremental form. Another route is given by incremental fitting a parabola to sets of $(2 d+1)$ successive data points, where $d$ is usually equal to $1,2,3$, or 4 [38]. Last, it is possible to use a best smooth curve through $a(N)$ data and taking gradients from the interpolation [39-41]. This route was followed by the authors in a previous study [28]. The present study aims at introducing an automated procedure that considers the proposed propagation law inside the DIC analysis.

In the following, actual values of $a$ and $\Delta K$ are estimated together with an additive noise $\eta_{a}$ and $\eta_{K}$. The measured values will bear an ${ }_{m}$ subscript. As discussed in Ref. [12], no bias is expected and hence their statistical average vanishes $\langle\eta\rangle=0$. At different instants of time, the noise is assumed to be uncorrelated. Consequently, the noise is characterized by its covariance ma$\operatorname{trix} C_{i j}$

$$
\left\langle\eta_{i}(N) \eta_{j}\left(N^{\prime}\right)\right\rangle=C_{i j} \delta\left(N-N^{\prime}\right)
$$

where $\langle\bullet\rangle$ denotes the average over the load history. The estimate of the SIFR $\Delta K$ is performed through a technique that involves "super-singular" modes in Williams' expansion $[13,32,28]$. As a result, the dominant contribution of an ill-positioning of the crack tip on the stress intensity factor is canceled. Only a second order term remains. Therefore the cross-correlation $C_{a K}$ between $a$ and $\Delta K$ noise vanishes. Measured $a$ and $\Delta K$ as functions of the number of 
cycles is thus expected to obey

$$
a(N)=a\left(N_{0}\right)+\eta_{a}(N)+\int_{N_{0}}^{N} \Psi\left(\Delta K(M)-\eta_{K}(M)\right) \mathrm{d} M
$$

The difficulty is to design a robust method to evaluate at best the crack growth law, $\Psi$. The unknowns are the parameters entering in the expression of $\Psi$ as well as the initial position $a\left(N_{0}\right)$ of the crack, which can be added to the vector of unknowns $\alpha_{i}$.

\subsection{Procedure 1}

Starting with an initial guess of the unknown parameters based on I-DIC (e.g., as performed in Ref. [28]), at each instant of time $N$, the $\operatorname{SIFR} \Delta K_{m}(N)$ is estimated. A predicted crack tip position $a_{\text {predict }}$ at the next time step is obtained based on the current crack tip position. This position is proposed to the I-DIC analysis (a needed ingredient to run an integrated DIC code since the crack tip position has to be known in Williams' series (3)), and $\Delta K_{m}$ is evaluated at this new position. This can be seen as an explicit integration in time, where $\Delta K_{m}$ is measured rather than computed. Since the I-DIC code returns an estimated position for the crack tip (i.e., by canceling out amplitude $\left.\omega_{-1}\right), a_{\text {eval }}(N)$, an error term is computed from the difference $\Delta a(N)=a_{\text {eval }}(N)-a_{\text {predict }}(N)$. This error is stored but not used at this stage.

After running this procedure for all images recorded during the test, the error series $\Delta a(N)$ is now used to correct the unknown parameters. This is done as a minimization of the difference between the measured and predicted crack tip positions at all instants of time globally. This non-linear minimization problem is solved iteratively, where each iteration is based on a first order perturbation of $\Psi$ with respect to the unknowns $\alpha_{i}$. The crack tip position modification 
$b_{i}(N)$ is computed from a mere differentiation of Equation (10) by ignoring noise

$$
b_{i}(N)=\int_{N_{0}}^{N} \frac{\partial \Psi\left(\Delta K_{m}(M)\right)}{\partial \alpha_{i}} \mathrm{~d} M
$$

If the initial position of the crack is added to the list of unknowns as $\alpha_{n+1}$, then

$$
b_{n+1}(N)=1
$$

The error $\Delta a(N)$ is then projected (in the least-squares sense) onto the set of functions $b_{i}(N)$ from a minimization of

$$
\mathcal{T}\left(\beta_{i}\right)=\sum_{N}\left(\Delta a(N)-\sum_{i} \beta_{i} b_{i}(N)\right)^{2}
$$

The coefficients $\beta_{i}$ are then considered as estimates of the correction of the trial parameters $\alpha_{i}$, which are updated through

$$
\alpha_{i} \longleftarrow \alpha_{i}+\beta_{i}
$$

These values of $\alpha_{i}$ are used to calculate a new $a(N)$ series by direct integration of Equation (7), and then a new $b_{i}(N)$. This incremental correction of the parameters is iterated until the difference between the last two $a(N)$ becomes small. Once a new law has been obtained, a global step of the proposed method has been completed.

The same step is repeated up to convergence of parameters to steady values. This algorithm accounts for the uncertainty in the crack tip position because of the least-squares formulation, which is the most appropriate to a Gaussian white noise. However, the uncertainty in $\Delta K$ yields a non-linear term, and hence it produces a bias on the evaluation of the best parameters. This systematic error is proportional to the variance of the uncertainty on $\Delta K_{m}$ and the weight term equal to the time integral of the second order derivative of $\Psi$. 


\subsection{Procedure 2}

The difficulty observed for the previous procedure (i.e., biased estimates) is difficult to by-pass without additional assumptions. As the loading history $\Delta K(N)$ is assumed to be arbitrary and unknown, there is no way to properly separate signal from noise in $\Delta K_{m}(N)$. However, it may be assumed that the actual value $\Delta K(N)$ (without noise) has a smooth change with time. This allows for a more faithful strategy illustrated in Figure 1.

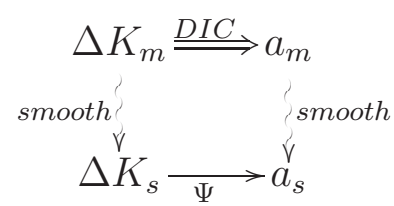

Fig. 1. Schematic diagram of the relationship between measured quantities $\Delta K_{m}$ and $a_{m}$ and intermediate smooth evolutions $\Delta K_{s}$ and $a_{s}$.

The idea is to introduce two intermediate functions $\Delta K_{s}(N)$ and $a_{s}(N)$, which are related by integration of the crack growth law, and such that both quantities are smooth versions of the experimental data. To construct the smooth signal $\Delta K_{s}(N)$, different routes may be followed. For instance, a space of smooth functions may be chosen such as a polynomial basis [39] for $\Delta K_{s}$. Conversely, rapid variations in $\Delta K_{s}(N)$ may be penalized through, say, a quadratic norm of the second derivative, and in both cases secure the proximity of $\Delta K_{s}$ and $\Delta K_{m}$ with a quadratic difference to be minimized. For instance, a functional such as

$$
\begin{aligned}
W_{1}= & \sum_{N}\left(\Delta K_{m}(N)-\Delta K_{s}(N)\right)^{2} \\
& +w \sum_{N}\left(\Delta K_{s}(N+\delta N)-2 \Delta K_{s}(N)+\Delta K_{s}(N-\delta N)\right)^{2}
\end{aligned}
$$

minimized over $\Delta K_{s}$ is a simple way to filter $\Delta K_{m}$, where $\delta N$ is the sampling 
interval. Note that this functional contains a smoothing parameter $w$ that sets a time scale below which the function is smooth and above which it sticks to the data. For a very large level $w, \Delta K_{s}$ tends to a linear law with $N$, which is nothing but a best linear fit through the data $\Delta K_{m}$. For a Gaussian white noise acting on $\Delta K_{m}$ this procedure is a very powerful way of extracting the relevant signal from noise.

For a set of parameters $\alpha_{i}, a_{s}$ is obtained from the time integration of $\Delta K_{s}$. Finally, to close the loop, the same strategy as in procedure 1 is kept, namely, incremental signals $b_{i}(N)$ are constructed from $\Delta K_{s}$ from variations of the parameters $\alpha_{i}$, and the difference $a_{m}-a_{s}$ is decomposed over this set of smooth functions $b_{i}$ to estimate the proper modification of the assumed $\alpha$ parameters for the best match. As above mentioned this projection is already written in an optimal way for a Gaussian white noise.

Not only is this procedure expected to account for the uncertainty both for $\Delta K$ and $a$, but it also naturally provides an estimate of "admissible" (in the sense of fulfilling the propagation law, being smooth, and constrained by image registration) signals $\Delta K_{s}$ and $a_{s}$. The practical implementation of this approach is very close to the previous one, but rather than using the actual measurements of $\Delta K_{m}$, the SIFR is first filtered prior to the application of procedure 1. To initiate the procedure, the first step is performed with no filter as in procedure 1 .

\section{Test on artificial cases}

To test the proposed methodology, a series of data obeying a known propagation law was generated together with a time varying stress intensity factor 
range and including noise to a level representative of experimental cases. Figure 2 shows an example of series $a_{m}$ and $\Delta K_{m}$. One thousand such series have been generated and analyzed as if they were the experimental data in order to evaluate both systematic bias and standard uncertainty. The chosen law is with $A_{0}=2.5 \times 10^{-8}$ and $m_{0}=3.1$, when $a$ is expressed in $\mathrm{mm}$ and $\Delta K$ in $\mathrm{MPa} \sqrt{\mathrm{m}}$. The noise level is characterized by a standard deviation of $0.1 \mathrm{~mm}$ for $a$, and $0.2 \mathrm{MPa} \sqrt{\mathrm{m}}$ for $\Delta K$. All those values (parameters and uncertainties) are chosen to be representative of the test to be analyzed in the next section. They however allow for the generation of artificial data with a known law, and hence provide an evaluation of the uncertainty due to noise and the ability of the proposed procedure to deal with such noisy data.

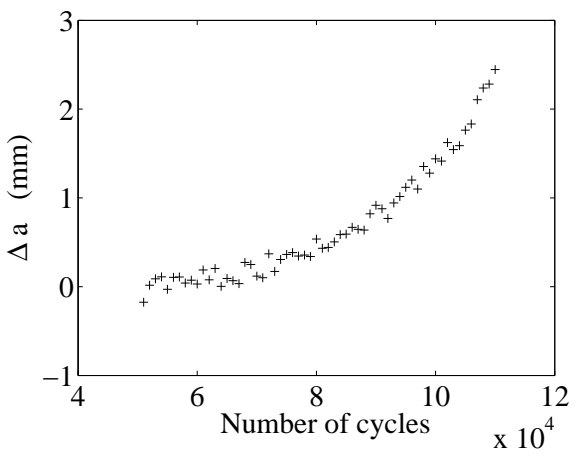

(a)

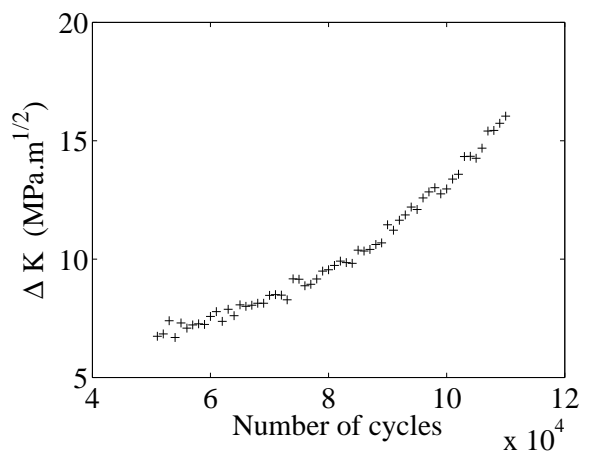

(b)

Fig. 2. Artificial data corresponding to the "measured" crack tip position $a_{m}$ (a) and SIFR $\Delta K_{m}$ (b) including noise.

As a reference, the first method used to evaluate the crack growth law, is the straightforward linear regression of the log-log plot of $\mathrm{d} a / \mathrm{d} N$ vs. $\Delta K$ as proposed in Ref. [37]. Such a plot is shown in Figure 3. It is observed that a very large scatter of points gives a rather unaccurate evaluation of the unknown 
parameters. The result over the 1000 random selections gives

$$
\begin{aligned}
& A / A_{0}=1.46 \pm 2.46 \\
& m / m_{0}=1.07 \pm 0.12
\end{aligned}
$$

The estimate of the amplitude shows a systematic error of about $50 \%$, and very large uncertainty levels. The exponent $m$ is determined with a much smaller bias and uncertainty, yet a $12 \%$ standard deviation is obtained.

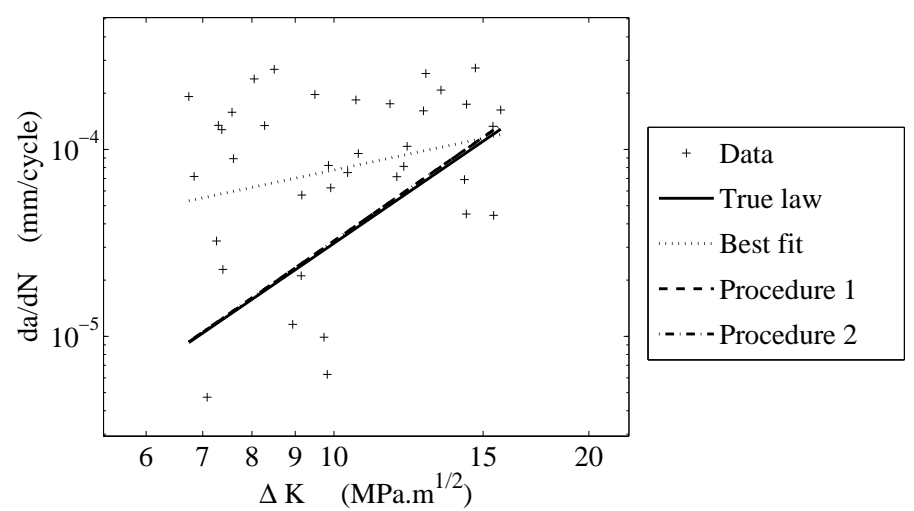

Fig. 3. Log-log plot of $\mathrm{d} a_{m} / \mathrm{d} N$ vs. $\Delta K_{m}$ for a typical artificial series including noise. The best fit is shown as a plain line, whereas the true imposed law is shown as a dotted line. The identified laws with procedures 1 and 2 are shown as dashed and dot-dashed lines. They essentially coincide with the true law. Both procedures proposed herein are able to yield consistent results whereas a direct fit does not.

The first procedure proposed above gives a much more satisfactory evaluation. Figure 4 shows the "measured" $a_{m}$ and the identified $a$ as a function of the reference (noiseless) a series. It is observed that the identification allows for the elimination of uncertainties in the series. 


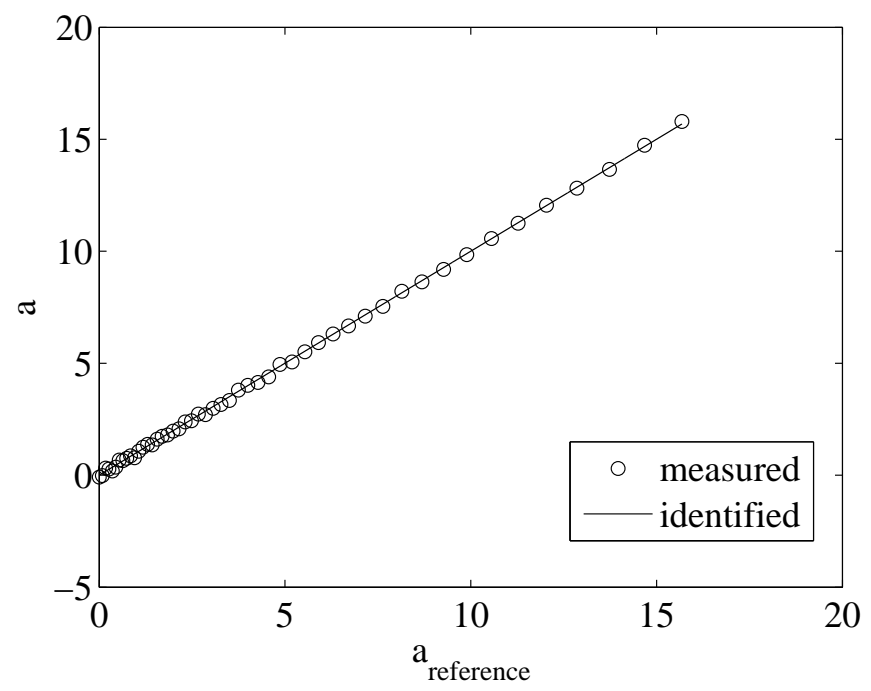

Fig. 4. Plot of the (artificial) "measured" crack tip position (symbols o) as a function of the noiseless position $a_{\text {reference }}$, for procedure 2 . The bold line shows the identified position, which cannot be distinguished from $a_{\text {reference }}$ (dotted line).

Statistics over the 1000 random selections provides the following estimates

$$
\begin{aligned}
& A / A_{0}=1.09 \pm 0.51 \\
& m / m_{0}=1.00 \pm 0.06
\end{aligned}
$$

Thus, both quantities appear as being essentially unbiased (in contrast to the former estimates), and a much lower uncertainty that is cut down to less than one tenth of its previously estimated value. Procedure 2 was also tested on the same series. Its results in terms of the unknown parameters are identical to those of the first procedure (Figure 3).

The main advantage of the second procedure over the first one is that it naturally provides filtered admissible $\Delta K(N)$ and $a(N)$ histories yet consistent with the identification procedure (i.e., not performed as a post-processing step). Other identification tests using a larger uncertainty shows a better stability of the exponent $m$ with procedure 2, but surprisingly a slight degra- 
dation of the systematic error and uncertainty on the prefactor $A$. However, the uncertainty remains always much larger then the systematic error, and the overall predictions of both methods remain quite close to each other, and allow for much more precise results than the straightforward linear regression (Figure 3).

\section{Analysis of a fatigue test}

Both procedures are now applied to a set of images of a crack propagation in a CCT sample made of a 0.3-mm thick sheet of commercially-pure titanium [28]. A tensile test along the rolling direction was performed. The dog-bone sample was monitored with a single camera (definition: $1022 \times 1024$ pixels, digitization: 12 bits) and a telecentric lens focused on the gauge length. The lens allows artifacts related to out-of-plane motions to be at least minimized if not completely canceled out. Figure 5(a) shows the true stress / true strain response when the mean stress is computed over the cross-sectional area of the sample, and the strains averaged over the whole region of interest.

By using an optical microscope the microstructure of the material was revealed. An average equiaxed grain size of the order of $20 \mu \mathrm{m}$ is observed (Figure $5(\mathrm{~b}))$. The main mechanical and microstructural features of the material are listed in Table 1.

A fatigue test is performed at constant load ratio $R=0.1$ with a $10-\mathrm{Hz}$ frequency. Pictures of the area of interest are shot every thousand cycles, at minimum and maximum load level. The maximum level corresponds to a remote stress about half the yield stress of the studied alloy (Table 1). As the goal of these procedures is to minimize the error $\Delta a(N)$ defined in Equation (6) 


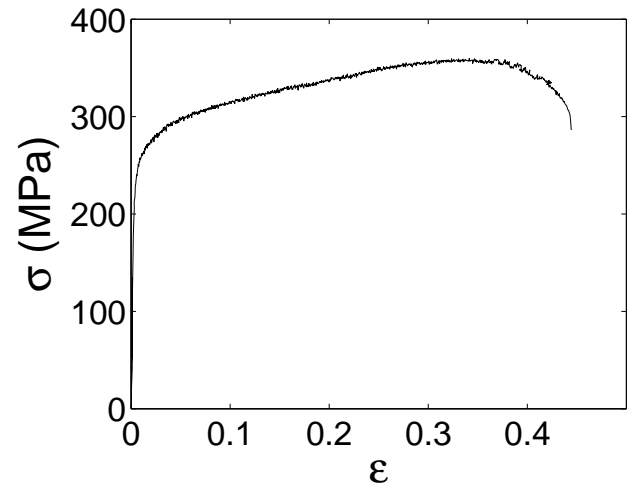

(a)

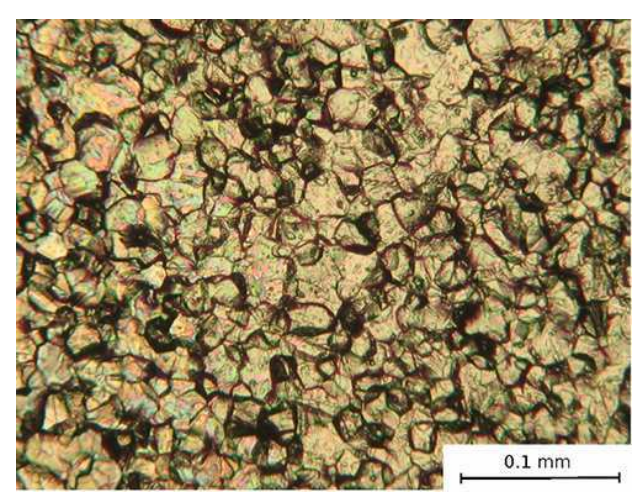

(b)

Fig. 5. True stress / true strain response of commercially-pure titanium tested in the rolling direction (a). Microstructure of the material (b).

Table 1

Mechanical and microstructural properties of commercially-pure titanium alloy

\begin{tabular}{|l|c|}
\hline Yield stress & $210 \mathrm{MPa}$ \\
Young's modulus & $100 \mathrm{GPa}$ \\
Poisson's ratio & 0.33 \\
Grain size & $20 \mu \mathrm{m}$ \\
\hline
\end{tabular}

in order to identify the best parameters $\alpha_{i}$, its variations are analyzed to assess the convergence of the algorithms

$$
\xi(i t)=\sqrt{\frac{1}{N} \sum_{i t=1}^{N}(a(N, i t)-a(N, i t-1))^{2}}
$$

with it the number of iterations of the algorithm. According to the evolutions observed, the procedure is stopped when $\xi$ is less than a chosen quantity, for instance $\xi<1$ pixel (or $6 \mu \mathrm{m}$ ).

A measured displacement field is shown in Figure 6 for an intermediate stage of propagation. It is smooth since an integrated approach was used. Further, 
not the whole picture is considered in the analysis, but only a disk centered about the estimated crack tip position. The parameters of the mask are chosen

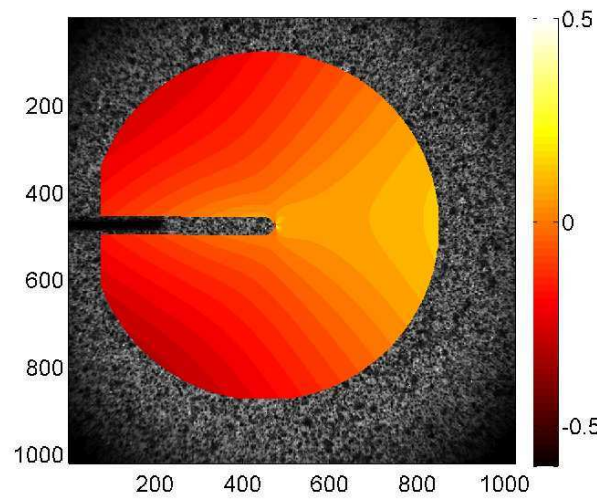

(a)

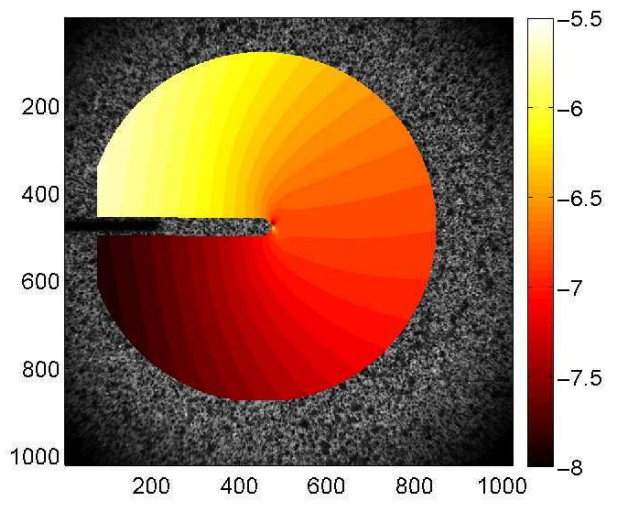

(b)

Fig. 6. Horizontal (a) and vertical (b) displacement fields for image pair 30. The displacements are expressed in pixels (1 pixel $\leftrightarrow 6.1 \mu \mathrm{m}$ ). The shape of the ROI is selected so that the local plastic zone in the vicinity of the crack tip is excluded. Since the crack front is not totally straight, an exclusion zone is also added. Last, since Williams' series is truncated for $n \leq 5$, an outer boundary is considered.

according to the domains of main existence of the Williams' series terms. To visualize the zones of dominance of the various terms, and to ensure that the analysis be performed over a zone of $K_{I}$-dominance, the amplitude of these terms is plotted along the ligament in Figure 7. It is concluded that the two dominant terms are those associated with mode I SIFR and T-stress range. Only the former will be analyzed hereafter. The interested reader will find results on the change of the T-stress component with the number of cycles in Ref. [28].

In contrast to the artificial case, it is observed that the direct implementation of the procedure diverges. Thus, to ensure stability and more precise results, 


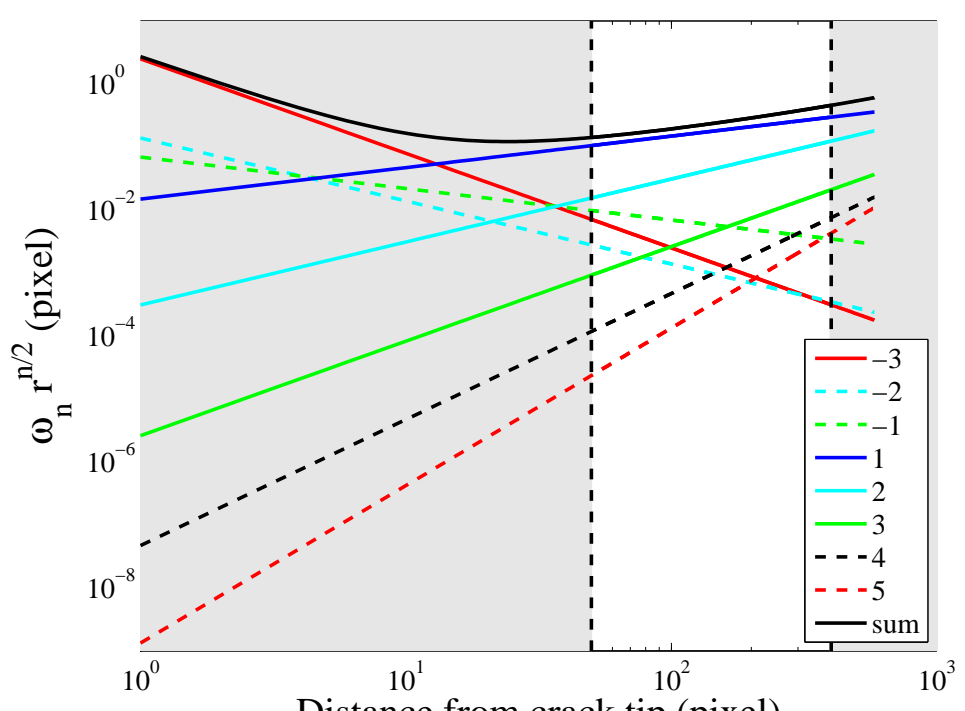

Fig. 7. Contributions of the terms of Williams' series for image pair 30 along the ligament. Vertical lines show the two radii $(1$ pixel $\leftrightarrow 6.1 \mu \mathrm{m})$. Abscissa 0 corresponds to the estimated crack tip location. The fields associated with $n=1$ (mode I SIFR) and $n=2$ (T-stress range) dominate the other ones.

an under-relaxation is implemented so that

$$
\alpha_{i} \longleftarrow \alpha_{i}+v \delta \alpha_{i}
$$

where $\delta \alpha_{i}$ is the change in the fatigue law parameters at the end of a global iteration loop. The ideal value of $0<v \leq 1$ can be determined for both methods as the one that minimizes the final error and the number of needed iterations at convergence. Figure 8 (a) displays results for several values of $v$. It is observed that as soon as $v$ is less than 0.9 , convergence is restored, and a subsequent decrease has only a modest impact on the convergence rate. In the following, a value of $v=0.5$ is chosen, and $\xi$ is plotted for both methods and both types of fatigue law in Figure 8(b). A value of $w=1$ is chosen for method 2 .

The use of a fatigue law with a threshold seems to help convergence and 


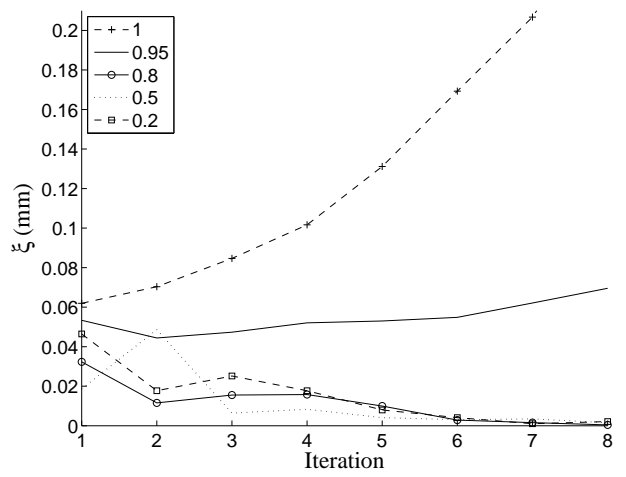

(a)

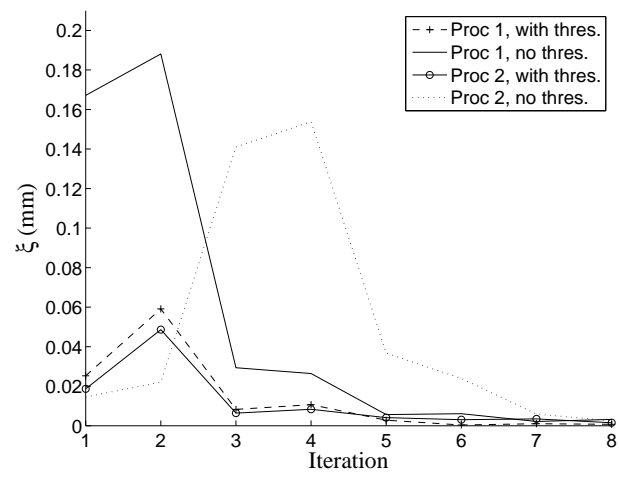

(b)

Fig. 8. (a) Plot of the error $\xi$ for the first iterations for several values of $v$ (method 2, Paris' law with threshold). (b) Error $\xi$ versus iteration number for both methods and both types of fatigue laws.

decrease slightly the final value of the residual error $\xi$. The lower residual is expected as the introduction of a threshold means that an additional degree of freedom is available to interpolate the data. However, this reduction is very modest, so that the uncertainty level on the threshold value is high. This is presumably due to the fact that the estimate of the threshold (when included) is roughly half the lowest value of $\Delta K$ observed in the experiment (see below). Experiments involving lower stress intensity factors should be performed to investigate this point. However, the easy convergence of the algorithm for this type of law allows us to be confident about its identification in more adapted cases.

It is to be stressed that the value of the residuals expressed in terms of standard deviation of crack tip position reaches very low values, about $30 \mu \mathrm{m}$, whereas the individual uncertainty on the position from a single image pair with an I-DIC analysis was evaluated to be about $100 \mu \mathrm{m}$ or 3 times larger [28]. This result shows that the uncertainty levels taken in the a priori analysis are overestimates to actual values. The fact that procedures 1 and 2 provided 
trustworthy estimates of the crack propagation law should also be expected with experimental data.

The raw data used in the present analysis are those given by an integrated approach to DIC. They were shown to lead to lower correlation residuals than those obtained with a standard DIC technique and subsequently postprocessed to evaluate fracture mechanics parameters [28]. The identified parameters are gathered in Table 2. They are compared with those of a direct fit of $d a / d N$ and $\Delta K$ data, and a fit of $a(N)$ and $\Delta K[28]$ used to initialize the two procedures introduced herein. An excellent consistency is observed between both proposed methods. To highlight the sensitivity to the type of law and the procedure followed, Figure 9 shows the differences between the former approaches and the new procedures. A last approach is based on a handbook solution [42]. When the position of the crack tip is known (i.e., estimated by I-DIC), the SIFR $\Delta K$ reads

$$
\Delta K_{a n a}=\frac{\Delta F \sqrt{\pi a}}{2 b c}\left[1-0.025\left(\frac{a}{c}\right)^{2}+0.06\left(\frac{a}{c}\right)^{4}\right] \sqrt{\frac{1}{\cos \left(\frac{\pi a}{2 c}\right)}}
$$

where $\Delta F$ is the applied load range, $b$ the thickness of the sample, $c$ its width, and $a$ the length of the center crack, which is the sum of the initial length and of the crack propagation increments. The propagation is assumed to be symmetric in the specimen. The estimate based on the closed-form solution (22) is not consistent with all the others. This result shows that the crack tip position has to be monitored very accurately but the SIFR also has to be assessed since it may depart from a handbook solution, for instance because propagation is not necessarily symmetric in a CCT experiment.

Figure 10 shows more precisely Paris' plot $\mathrm{d} a / \mathrm{d} N$ vs. $\Delta K$ with data points and identified laws for both types of propagation law and both procedures. 
Table 2

Estimated parameters of the fatigue crack propagation laws for five different approaches when $a$ is expressed in $\mathrm{mm}$ and $\Delta K$ in $\operatorname{MPa} \sqrt{\mathrm{m}}$.

\begin{tabular}{|l|c|c|c|c|c|}
\hline Identification & $A$ & $m$ & $B$ & $p$ & $\Delta K_{0}$ \\
Method & $\times 10^{-8}$ & & $\times 10^{-6}$ & & \\
\hline$d a / d N$ vs. $\Delta K_{\text {ana }}$ & 0.26 & 4.38 & & & \\
$d a / d N$ vs. $\Delta K_{\text {meas }}$ & 10.4 & 2.75 & 1.47 & 1.95 & 3.30 \\
$a(N)$ vs. $\Delta K_{\text {meas }}$ & 4.7 & 3.12 & 1.48 & 2.00 & 3.46 \\
Procedure 1 & 2.5 & 3.37 & 1.82 & 1.98 & 4.08 \\
Procedure 2 & 2.4 & 3.39 & 1.79 & 1.98 & 4.03 \\
\hline
\end{tabular}

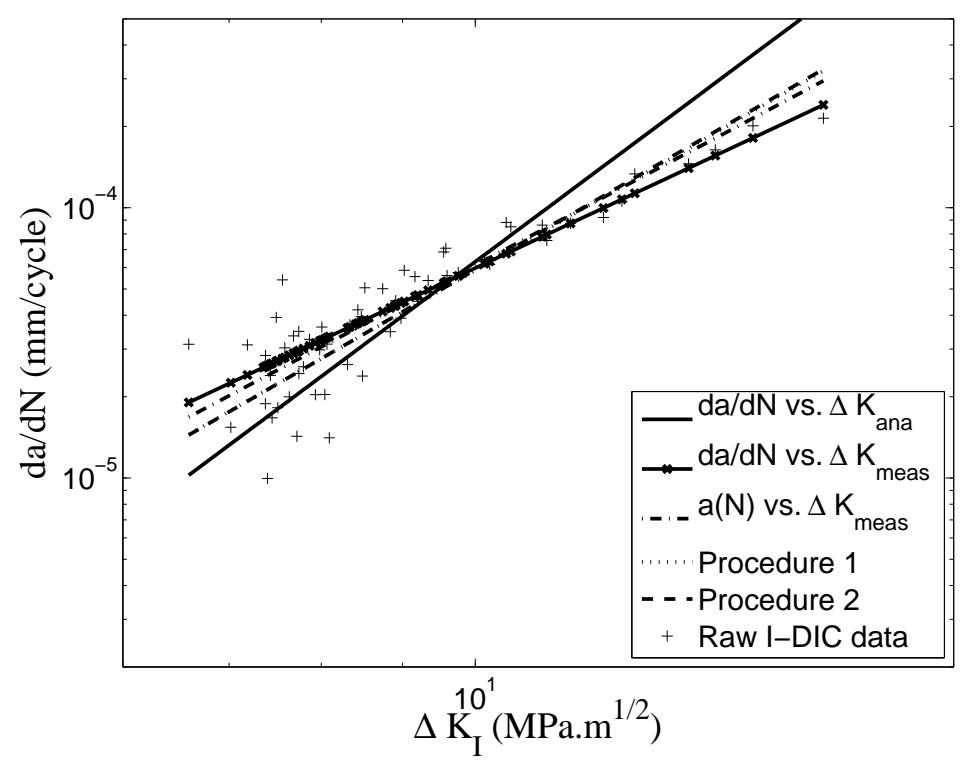

Fig. 9. Paris' plot for the five proposed methods: direct fit of $d a / d N$ and analytical $\Delta K$ estimate, direct fit of $d a / d N$ and measured $\Delta K$, fit of $a(N)$ and $\Delta K$ [28], procedures 1 and 2. The raw data are those measured by an integrated DIC approach [28]. All plots are for a Paris' law with no threshold. 
It is worth noting that the initialization values for procedures 1 and 2 were

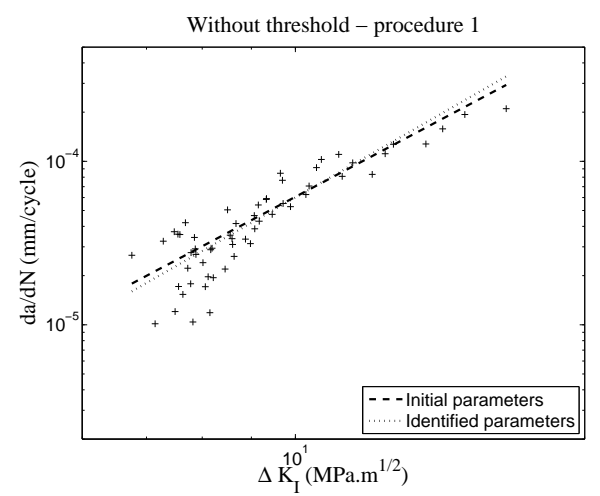

(a)

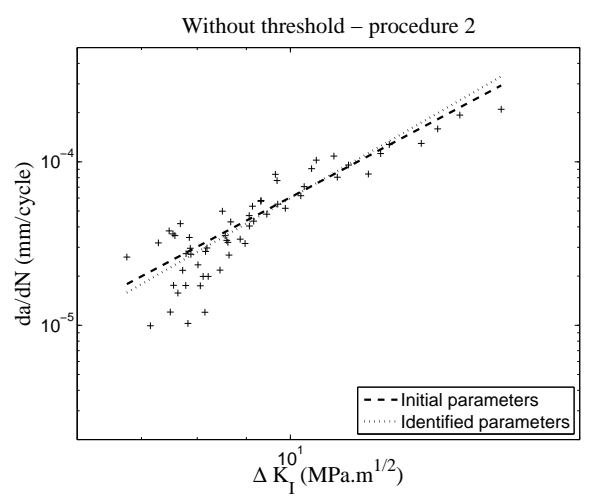

(c)

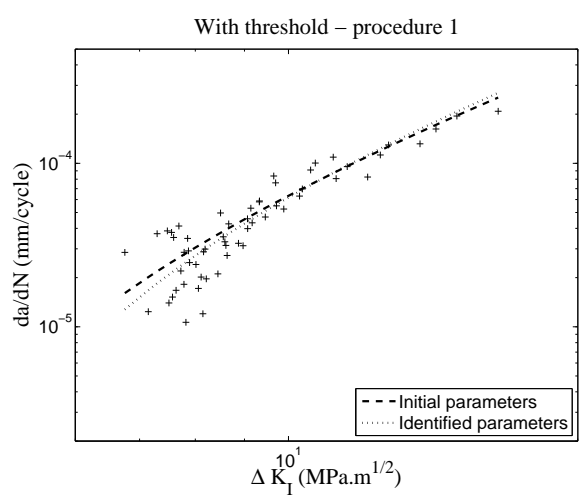

(b)

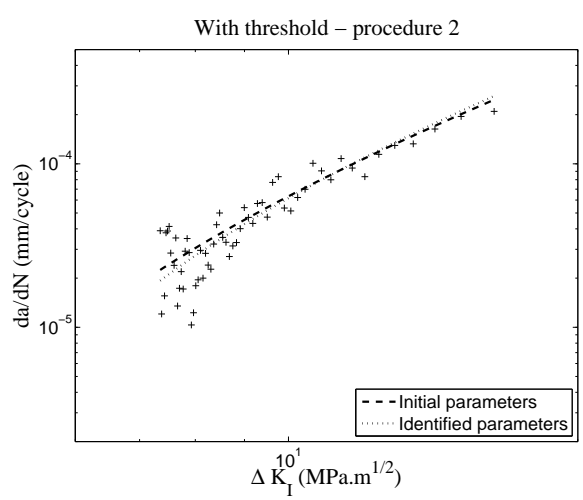

(d)

Fig. 10. Plot of the Paris' and modified Paris' law for the initialization values of the parameters and the final parameters. (a) Without threshold, procedure 1. (b) With threshold, procedure 1. (c) Without threshold, procedure 2. (d) With threshold, procedure 2 .

chosen based on a prior analysis of the same experiment using a $a(N)-\Delta K(N)$ fit [28]. Figure 10 displays the initial guess together with the identified one. Changes are small, yet they reveal significant in the evaluation of the residual error $\xi$. It is to be noted that the gray level correlation residuals $R(N)$ are around 2 percent of the gray level dynamic range anywhere in the image series, which is very low and allows for a fine determination of all terms in Williams' series, especially the first supersingular contribution that provides 
the predicted crack tip position $a_{\text {predict }}$.

The final error for each method is shown in Figure 11 for the crack tip position as a function of image number (i.e., with a fixed number of cycle increment). There are two remarkable features in these results. First, there is a high frequency fluctuation, which is of the order of $\pm 20 \mu \mathrm{m}$. This value corresponds to twice the grain size of the material. This observation suggests that there is an effect that may be related to the underlying microstructure of the material. Second, there is a low frequency component $(\approx 2 \mathrm{~mm})$ that is significantly larger than the grain size. This second effect is a systematic deviation from the assumed propagation law even though the crack propagation rate $d a / d N \geq 10^{-5} \mathrm{~mm} /$ cycle so that it is believed that Paris' regime is relevant [38]. These two effects could be quantified thanks to the chosen error indicator, and the accuracy of the proposed measurement technique allowing for crack tip positions to be assessed at resolutions less than the grain size of the material.

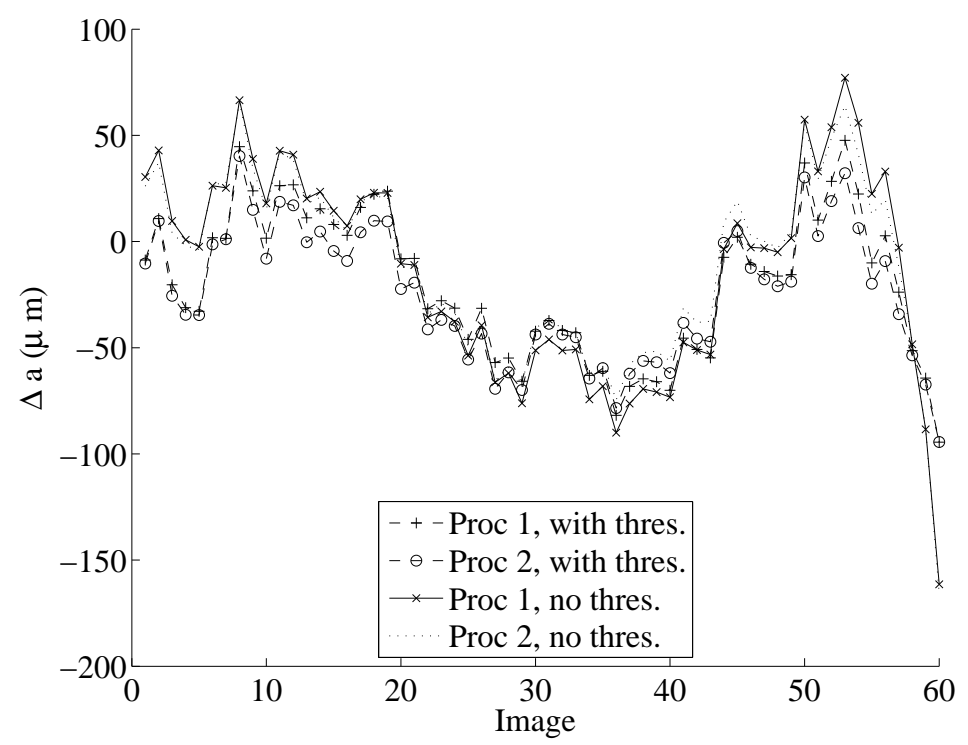

Fig. 11. Plot of the quantity $\Delta a$ for the final values of the parameters for each method and the two propagation laws. 
The integrated DIC analysis provides more information than the stress intensity factor range and crack tip position. $T$-stress, or size of the plastic zone also can be evaluated from this analysis [28]. As has been suggested in the literature, these additional features may contribute to the crack propagation law. Although an enriched form of Paris' law incorporating these parameters has not been tried in the complete analysis, the change of those parameters with the number of cycle does not correlate significantly with the final error (i.e., correlation coefficients of the order of 0.1 to 0.15 ). This result suggests that such an enrichment would not enhance much the quality of the identified law (apart from the trivial benefit of having additional degrees of freedom in the fitting procedure). This conclusion needs to be checked in the future, and is limited to the present experiment (material, microstructure and loading conditions) that may not highlight such additional dependencies.

\section{Summary}

The traditional way of identifying a fatigue crack propagation law starting from images available from the experiment would be to first resort to a DIC analysis of the displacement field, then to extract from it stress intensity factors and crack tip position by a first post-processing step, and finally to process the data to fit, say, a Paris' law by resorting to a second post-processing step. Each of these steps progressively reduces the available information, from pixels to displacements of zones of interest, then to SIFR and $a$ values, and finally to Paris' law parameters. These steps are projections from a basis to another one that is followed one way.

It was shown in earlier publications that the first two steps could be merged 
into the so-called integrated DIC, so that the estimate of $\Delta K$ (in particular) is confronted to the actual image directly. A significant benefit resulted from such a merger. In the present study, an additional integration is proposed so that the sought propagation law is evaluated directly from its ability to account for image evolutions. This procedure naturally incorporates the actual uncertainties attached to each image with respect to a specific kinematic basis (that of displacement fields associated with Williams' series) and crack tip position. As compared to traditional ways of addressing such an identification problem, the two-way exchange of information between the input (images) and output (crack propagation law) is the most striking difference. A clear benefit results from such a dialog. In particular, it was shown that the resolution of the identification procedure could achieve crack tip positioning three times less than what is usually required, namely in the $30 \mu \mathrm{m}$ range.

Two different techniques were introduced herein. For the analyzed experiment very close parameters were found. A clear distinction between the two procedures is still to be investigated, even though procedure 2 is theoretically able to deal with more difficult cases, i.e., with more noise.

Numerous perspective can be listed:

- The identification of constitutive laws where history matters (e.g., plasticity, viscoplasticity, viscoelasticity, damage) could be tackled from a series of images in a way similar to the presented approach. In a number of previous works, plasticity or damage behaviors have been addressed when based on a series of sequential treatment of data. The full dialog between constitutive law identification and images has not been tackled up to our knowledge aside from elasticity. A substantial gain in terms of accuracy and reliability is expected from such two-way approaches. 
- Three dimensional analyses, either based on surface or bulk measurements is most often needed. The present case was dedicated to a thin titanium sheet where a 2D description is not a strong limitation. However, in most cases, free surfaces induce a major alteration in the development of plasticity so that the applicability of a $2 \mathrm{D}$ analysis is questionable. In such a case, one could envision coupling a surface analysis to a full three dimensional modeling, or a full 3D analysis if say tomographic data is exploited as in refs. $[43,32,44,45]$.

- Control of an experiment from learning schemes based on identification and DIC is a very promising direction [46-48]. The methodology proposed in the present paper is one of the building bricks involved in such a goal.

\section{Acknowledgments}

It is a pleasure to acknowledge the support of the ANR project "RUPXCUBE" (ANR-09-BLAN-0009-01) and Région Ile de France ("FRESCORT" project).

\section{References}

[1] W. G. Clark and S. J. Hudack Jr., Variability in fatigue crack growth testing $J$. Test. Eval. 3 (1975) 454-476.

[2] C. J. Beevers, edt., The measurement of crack length and shape during fracture and fatigue, (EMAS, West Midlands (UK), 1980).

[3] S. J. Hudack Jr. and R. J. Bucci, edts., Fatigue crack growth emasurement and data analysis, (ASTM, Philadelphia, PA (USA), 1981), STP 738. 
[4] C. J. Beevers, edt., Advances in Crack Length Measurement, (EMAS, West Midlands (UK), 1982).

[5] K. J. Marsh, R. A. Smith and O. R. Ritchie, edts., Fatigue crack measurement: Technique and Applications, (EMAS, West Midlands (UK), 1991).

[6] ASTM Standard, E647-11: Standard Test Method for Measurement of Fatigue Crack Growth Rates, (ASTM International, West Conshohocken, PA (USA), 2003).

[7] H. Czichos, T. Saito and L. Smith, edts., Springer handbook of materials measurement methods, (Springer, 2006).

[8] R. Bowman, S. D. Antolovich and R. C. Brown, A demonstration of problems associated with crack closure measurement techniques, Eng. Fract. Mech. 31 [4] (1988) 703-712.

[9] M. A. Sutton, J.-J. Orteu and H. Schreier, Image correlation for shape, motion and deformation measurements: Basic Concepts, Theory and Applications, (Springer, New York, NY (USA), 2009).

[10] S. R. McNeill, W. H. Peters and M. A. Sutton, Estimation of stress intensity factor by digital image correlation, Eng. Fract. Mech. 28 [1] (1987) 101-112.

[11] J. Abanto-Bueno and J. Lambros, Investigation of crack growth in functionally graded materials using digital image correlation, Eng. Fract. Mech. 69 (2002) 1695-1711.

[12] S. Roux and F. Hild, Stress intensity factor measurements from digital image correlation: post-processing and integrated approaches, Int. J. Fract. 140 [1-4] (2006) 141-157.

[13] R. Hamam, F. Hild and S. Roux, Stress intensity factor gauging by digital image correlation: Application in cyclic fatigue, Strain 43 (2007) 181-192. 
[14] D. S. Dawicke and M. S. Sutton, CTOA and Crack-Tunneling Measurements in Thin Sheet 2024-T3 Aluminum Alloy, Exp. Mech. 34 (1994) 357-368.

[15] M. A. Sutton, W. Zhao, S. R. McNeill, J. D. Helm, R. S. Piascik and W. T. Riddel, Local crack closure measurements: Development of a measurement system using computer vision and a far-field microscope, in: Advances in fatigue crack closure measurement and analysis: second volume, STP 1343, R. C. McClung and J. C. Newman Jr., edts., (ASTM, 1999 145-156.

[16] W. T. Riddel, R. S. Piascik, M. A. Sutton, W. Zhao, S. R. McNeill and J. D. Helm, Determining fatigue crack opening loads from near-crack-tip displacement measurements, in: Advances in fatigue crack closure measurement and analysis: second volume, STP 1343, R. C. McClung and J. Newman, J.C., eds., (ASTM, 1999 157-174).

[17] J. Rupil, S. Roux, F. Hild and L. Vincent, Fatigue microcrack detection with digital image correlation, J. Strain Analysis 46 [6] (2011) 492-509.

[18] M. L. Williams, On the stress distribution at the base of a stationary crack, ASME J. Appl. Mech. 24 (1957) 109-114.

[19] G. R. Irwin, Analysis of the Stresses and Strains near the End of a Crack Traversing a Plate, ASME J. Appl. Mech. 24 (1957) 361-364.

[20] S. G. Larsson and A. J. Carlsson, Influence of Non-Singular Stress Terms and Specimen Geometry on Small Scale Yielding at Crack-Tip in Elastic-Plastic Materials, J. Mech. Phys. Solids 21 (1973) 263-277.

[21] J. R. Rice, Limitations to the Small Scale Yielding Approximation for Crack-Tip Plasticity, J. Mech. Phys. Solids 22 (1974) 17-26.

[22] K. Tanaka, T. Hoshide, A. Yamada and S. Taira, Fatigue crack propagation in biaxial stress fields, Fat. Fract. Eng. Mat. Struct. 2 (1979) 181-194. 
[23] H. Youshi, M. W. Brown and K. J. Miller, Fatigue crack growth from a circular notch under high levels of biaxial stress, Fat. Eng. Mater. Struct. 15 [12] (1992) $1185-1197$.

[24] J. Tong, T-stress and its implications for crack growth, Eng. Fract. Mech. 69 (2002) 1325-1337.

[25] I. C. Howard, A method of estimating biaxial fatigue growth rates, Fat. Fract. Eng. Mat. Struct. 3 (1981) 265-270.

[26] F. A. McClintock, Discussion to C. Lairds paper The influence of metallurgical microstructure on the mechanisms of fatigue crack propagation, Proceedings Fatigue crack propagation, (Philadelphia, PA (USA), 1967), 170-174.

[27] M. J. Maleski, M. S. Kirugulige and H. V. Tippur, A Method for Measuring Mode I Crack Tip Constraint Under Static and Dynamic Loading Conditions, Exp. Mech. 44 [5] (2004) 522-532.

[28] F. Mathieu, F. Hild and S. Roux, Identification of a crack propagation law by digital image correlation, Int. J. Fat. 36 (2012) 146-154.

[29] J. Réthoré, S. Roux and F. Hild, Optimal and noise-robust extraction of Fracture Mechanics parameters from kinematic measurements, Eng. Fract. Mech. 78 [9] (2011) 1827-1845.

[30] P. C. Paris, M. P. Gomez and W. P. Anderson, A Critical Analysis of Crack Propagation Laws, The Trend in Engineering 13 (1961) 9-14.

[31] P. C. Paris and F. Erdogan, A critical analysis of crack propagation laws, J. Basic Eng. 85 [4] (1963) 528-534.

[32] S. Roux, J. Réthoré and F. Hild, Digital Image Correlation and Fracture: An Advanced Technique for Estimating Stress Intensity Factors of 2D and 3D Cracks, J. Phys. D: Appl. Phys. 42 (2009) 214004. 
[33] C. Henninger, S. Roux and F. Hild, Enriched kinematic fields of cracked structures, Int. J. Solids Struct. 47 (2010) 3305-3316.

[34] W. Elber, Fatigue Crack Closure under Cyclic Tension, Eng. Fract. Mech. 2 (1970) 37-45.

[35] W. Elber, The Significance of Fatigue Crack Closure, in: Damage Tolerance in Aircraft Structures (ASTM, STP 486, Philadelphia, 1971 230-242.

[36] J. R. Mohanty, B. B. Verma and P. K. Ray, Determination of fatigue crack growth rate from experimental data: A new approach, Int. J. Microstr. Mat. Prop. 5 [1] (2010) 79-87.

[37] B. Mukherjee, A note on the analysis of fatigue crack growth data, Int. J. Fract. 8 (1972) 449-451.

[38] ASTM, E647-08: Standard test method for measurement of fatigue crack growth rates, (ASTM, West Conshohocken, PA (USA), 2008).

[39] K. B. Davies and C. E. Feddersen, Evaluation of fatigue-crack growth rates by polynomial curve fitting, Int. J. Fract. 9 (1973) 116-118.

[40] R. A. Smith, The determination of fatigue crack growth rates from experimental data, Int. J. Fract. 9 (1973) 352-355.

[41] J. Polak and Z. Knesl, On the fatigue crack growth rate evaluation from experimental data, Int. J. Fract. 11 (1975) 693-96.

[42] H. Tada, P. C. Paris and G. R. Irwin, The Stress Analysis of Cracks Handbook, (Del Research, St Louis (USA), 1985).

[43] N. Limodin, J. Réthoré, J.-Y. Buffière, A. Gravouil, F. Hild and S. Roux, Crack closure and stress intensity factor measurements in nodular graphite cast iron using 3D correlation of laboratory X ray microtomography images, Acta Mat. 57 [14] (2009) 4090-4101. 
[44] N. Limodin, J. Réthoré, J.-Y. Buffière, F. Hild, S. Roux, W. Ludwig, J. Rannou and A. Gravouil, Influence of closure on the 3D propagation of fatigue cracks in a nodular cast iron investigated by X-ray tomography and 3D Volume Correlation, Acta Mat. 58 [8] (2010) 2957-2967.

[45] J. Rannou, N. Limodin, J. Réthoré, A. Gravouil, W. Ludwig, M.-C. BaïettoDubourg, J.-Y. Buffière, A. Combescure, F. Hild and S. Roux, Three dimensional experimental and numerical multiscale analysis of a fatigue crack, Comp. Meth. Appl. Mech. Eng. 199 (2010) 1307-1325.

[46] X. Fayolle, S. Calloch and F. Hild, Controlling testing machines with digital image correlation, Exp. Tech. 31 [3] (2007) 57-63.

[47] X. Fayolle, Correli ${ }^{F I C}$ : programme de pilotage d'essais asservis sur un facteur d'intensité des contraintes, (MSc report, CNAM Paris, 2008).

[48] E. Durif, M. Fregonese, J. Réthoré and A. Combescure, Development of a Digital Image Correlation controlled fatigue crack propagation experiment, in: F. Brémand, ed., Proceedings ICEM 14, (EPJ Web of Conferences, 2010). 


\section{List of Figures}

1 Schematic diagram of the relationship between measured quantities $\Delta K_{m}$ and $a_{m}$ and intermediate smooth evolutions $\Delta K_{s}$ and $a_{s}$.

2 Artificial data corresponding to the "measured" crack tip position $a_{m}$ (a) and SIFR $\Delta K_{m}$ (b) including noise.

3 Log-log plot of $\mathrm{d} a_{m} / \mathrm{d} N$ vs. $\Delta K_{m}$ for a typical artificial series including noise. The best fit is shown as a plain line, whereas the true imposed law is shown as a dotted line. The identified laws with procedures 1 and 2 are shown as dashed and dot-dashed lines. They essentially coincide with the true law. Both procedures proposed herein are able to yield consistent results whereas a direct fit does not.

4 Plot of the (artificial) "measured" crack tip position (symbols

○) as a function of the noiseless position $a_{\text {reference, }}$, for procedure 2. The bold line shows the identified position, which cannot be distinguished from $a_{\text {reference }}$ (dotted line).

5 True stress / true strain response of commercially-pure titanium tested in the rolling direction (a). Microstructure of the material (b). 
6 Horizontal (a) and vertical (b) displacement fields for image pair 30. The displacements are expressed in pixels (1 pixel $\leftrightarrow 6.1 \mu \mathrm{m}$ ). The shape of the ROI is selected so that the local plastic zone in the vicinity of the crack tip is excluded. Since the crack front is not totally straight, an exclusion zone is also added. Last, since Williams' series is truncated for $n \leq 5$, an outer boundary is considered.

7 Contributions of the terms of Williams' series for image pair 30 along the ligament. Vertical lines show the two radii (1 pixel $\leftrightarrow 6.1 \mu \mathrm{m}$ ). Abscissa 0 corresponds to the estimated crack tip location. The fields associated with $n=1$ (mode I SIFR) and $n=2$ (T-stress range) dominate the other ones.

8 (a) Plot of the error $\xi$ for the first iterations for several values of $v$ (method 2, Paris' law with threshold). (b) Error $\xi$ versus iteration number for both methods and both types of fatigue laws.

9 Paris' plot for the five proposed methods: direct fit of $d a / d N$ and analytical $\Delta K$ estimate, direct fit of $d a / d N$ and measured $\Delta K$, fit of $a(N)$ and $\Delta K[28]$, procedures 1 and 2. The raw data are those measured by an integrated DIC approach [28]. All plots are for a Paris' law with no threshold. 
10 Plot of the Paris' and modified Paris' law for the initialization values of the parameters and the final parameters. (a) Without threshold, procedure 1. (b) With threshold, procedure 1. (c) Without threshold, procedure 2. (d) With threshold, procedure 2 .

11 Plot of the quantity $\Delta a$ for the final values of the parameters for each method and the two propagation laws. 